\title{
THE
}

$1-1-1986$

\section{Gap of the Linear Spin-1 Heisenberg Antiferromagnet: A Monte Carlo Calculation}

\author{
M. P. Nightingale \\ University of Rhode Island, nightingale@uri.edu
}

H. W.J. Blöte

Follow this and additional works at: https://digitalcommons.uri.edu/phys_facpubs

Terms of Use

All rights reserved under copyright.

\section{Citation/Publisher Attribution}

Nightingale, M. P., \& Blöte, H. W. J. (1986). Gap of the linear spin-1 Heisenberg antiferromagnet: A Monte Carlo calculation. Physical Review B, 33(1), 659-661. doi:

Available at: http://dx.doi.org/10.1103/PhysRevB.33.659

This Article is brought to you for free and open access by the Physics at DigitalCommons@URI. It has been accepted for inclusion in Physics Faculty Publications by an authorized administrator of DigitalCommons@URI. For more information, please contact digitalcommons-group@uri.edu. 


\title{
Gap of the linear spin-1 Heisenberg antiferromagnet: A Monte Carlo calculation
}

\author{
M. P. Nightingale \\ Department of Physics, University of Rhode Island, Kingston, Rhode Island 02881 \\ H. W. J. Blöte \\ Laboratorium voor Technische Natuurkunde, Lorentzweg 1, Postbus 5046, 2600 GA Delft, The Netherlands
}

(Received 15 October 1985)

\begin{abstract}
We have performed Monte Carlo calculations of the energies of several low-lying energy states of onedimensional, spin-1 Heisenberg antiferromagnets with linear sizes up to $n=32$. Our results support Haldane's prediction that a gap exists in the excitation spectrum for $n \rightarrow \infty$.
\end{abstract}

Haldane's prediction ${ }^{1}$ that antiferromagnetic Heisenberg chains have a gap in their energy spectrum for integral, but not for half-integral, spin values has been studied quite extensively recently. Results obtained by finite-size scaling methods tend to support the existence of a gap for spin-1 chains. However, the reliability of the numerical evidence is controversial, in the sense that it has been questioned whether the asymptotic regime of size dependence had been reached.

As it turns out, the earliest calculations ${ }^{2,3}$ on finite spin-1 chains provide an indication of a gap. The ground-state energy per spin was observed to converge with chain length $n$ more rapidly than $n^{-2}$. This is significant, since finite-size scaling would predict exponential convergence if a gap were present. ${ }^{4}$ In other words, the $n^{-2}$ size dependence, which is found for spin- $\frac{1}{2}$ chains and which is a signature of a spectrum without a gap, is absent for spin-1 chains.

A more direct check of Haldane's conjecture was provided by the work of Botet and co-workers, who calculated the gap of finite spin-1 chains up to $n=12 .^{5}$ They concluded that a gap does exist for $n \rightarrow \infty$, a result corroborated by subsequent work of Glaus and Schneider. ${ }^{6}$ This conclusion, however, was criticized by Bonner and Müller, and independently by Sólyom and Ziman. ${ }^{7}$ The spin- $\frac{1}{2}$ and spin-1 chains, when subjected to the same analyses, were found to behave quite similarly, while in fact it is known rigorously that a gap is absent for spin $\frac{1}{2} .^{8}$ According to Bonner and Müller, finite-size results for lengths up to as large as $n=30$ might be required to find the true asymptotic finite-size behavior.

Finally, Sólyom ${ }^{9}$ and Chui and $\mathrm{Ma}^{9}$ proposed phase diagrams of spin-1 Heisenberg antiferromagnets in disagreement with Haldane's conjecture.

To clarify matters, we have performed a Monte Carlo calculation of energy levels and gaps for spin-1 antiferromagnetic Heisenberg chains of lengths $16,20,24$, and 32, considerably larger than previously investigated.

In terms of the $z$ component of spin $s_{i}^{z}$ and the usual ladder operators $s_{i}^{+}$and $s_{i}^{-}$at site $i$, the Heisenberg Hamiltonian reads

$$
\mathscr{H}=-J \sum_{i=1}^{n}\left[\frac{1}{2}\left(s_{i}^{+} s_{i+1}^{-}+s_{i}^{-} s_{i+1}^{+}\right)+s_{i}^{z} s_{i+1}^{z}\right],
$$

with periodic boundary conditions, and $J<0$ in the antiferromagnetic case. Denoting by $|i\rangle,|j\rangle, \ldots$ the $3^{n}$ basis states in which the $s_{i}^{z}$ are diagonal, we introduce a matrix $\underline{H}$ with elements

$$
\begin{aligned}
& H_{i i}=(\langle i|\mathscr{P}| i\rangle-n) / J, \\
& H_{i j}=-\langle i|\mathscr{H}| j\rangle / J, \quad(i \neq j) .
\end{aligned}
$$

The matrix $\underline{H}$ has no negative elements, and its eigenvalues $\lambda$ are linearly related to those of $\mathscr{H}$; the latter equal $(\lambda-n) J .^{3}$ Furthermore, $\underline{H}$ is block diagonal; each block is characterized by the eigenvalue $S$ of $S^{z}$, the $z$ component of the total spin. The dominant eigenvalues of these blocks are denoted by $\lambda_{S}^{(n)}$ and the corresponding eigenvectors by $\mathbf{u}_{S}$, where spin and size indices are sometimes suppressed. The lowest levels $E_{S}^{(n)}$ of $\mathscr{P}$ are related to the corresponding eigenvalues of $\underline{H}$ by $\lambda_{S}^{(n)}=E_{S}^{(n)} / J+n$. The ground state and first excited state have $S=0$ and $S=1$, respectively, in systems up to $n=14,3,10$ and there are no indications that this should be any different for the spectra of longer chains.

The dominant eigenvalues of $\underline{H}$ were found by employing a simplified version of the Green's-function Monte Carlo method: ${ }^{11}$ a stochastic implementation of direct iteration. In the basic relation, which produces the leading eigenvector for large $k$,

$$
\mathbf{v}^{(k+1)}=\underline{H} \cdot \mathbf{v}^{(k)},
$$

the vectors are replaced by stochastic vectors $\mathbf{N}^{(k)}$ with components that are the integer-valued, stochastic variables $N_{i}{ }^{(k)}$. The latter are realized in subsequent generations of random walkers, each of which can be in any one of the basis states. For the $k$ th generation $N_{i}^{(k)}$ is the number of random walkers that are in state $|i\rangle$. To implement the matrix multiplication, one generation of random walkers is transformed into the next as follows. We write

$$
\underline{H}=\underline{H}^{(1)}+\underline{H}^{(2)} \text {, }
$$

where $\underline{H}^{(1)}$ contains the diagonal elements of $\underline{H}$ and $\underline{H}^{(2)}$ the off-diagonal ones. ${ }^{12}$ Now $\underline{H}^{(m)}(m=1,2)$ is written as

$$
\underline{H}^{(m)}=f_{k} \underline{C}^{(m)} \cdot \underline{D}^{(m)},
$$

where $f_{k}$ is a running estimate of the current leading eigenvalue of $\underline{H} . \underline{D}^{(m)}$ is a diagonal matrix such that the sum of the elements in each column of $\underline{C}^{(m)}$ equals one. Multiplying $\mathbf{N}^{(k)}$ by $\underline{H}$ stochastically is done in two parallel steps (corresponding to $m=1,2$ ) of two steps each [corresponding to $C^{(m)}$ and $D^{(m)}$. Each random walker in state $|i\rangle$ is replaced independently by $\left[D_{i i}\right]+1$ random walkers in the same state, with probability $D_{i i}-\left[D_{i i}\right]$, and by $\left[D_{i i}\right]$ random 
walkers otherwise, where $[r]$ is the integer part of a real number $r$. In this way, each random walker will be replaced by $D_{l l}$ of such on average.

Next, the multiplication by $\underline{C}^{(m)}$, which is nontrivial only for $m=2$, is simulated using the fact that $\underline{C}^{(m)}$ is a Markov matrix where $C_{i j}^{(m)}$ is the probability of a transition from state $j$ to $i$. The iterated stochastic process $\mathbf{N}^{(k)} \rightarrow \mathbf{N}^{(k+p)}$, by its very construction, is equivalent to multiplication by $\underline{H}^{p} / M_{k, p}$, where $M_{k, p}=\prod_{i=k}^{k+p^{-1}} f_{i}$, in the sense that the expectation value $\left\langle N^{(k+p)} M_{k, p}\right\rangle$ is given by

$$
\left\langle\mathbf{N}^{(k+p)} M_{k, p}\right\rangle=\underline{H}^{p} \cdot \mathbf{N}^{(k)} .
$$

As usual for direct iteration, for large $p,\left\langle\mathbf{N}^{(k+p)} M_{k, p}\right\rangle$ appropriately normalized converges to $\mathbf{u}$, the eigenvector corresponding to the dominant eigenvalue. One may take advantage of the adjustability of the $f_{k}$ to keep the numbers of random walkers $N^{(k)}$, the size of the $k$ th generation of random walkers, approximately constant at some target number $N$.

The dominant eigenvalue $\lambda$ can be estimated from a sequence of pairs of numbers $\left\langle N^{(k)}, f_{k}\right), k=0, \ldots, T$, in a variety of ways. One is tempted to use the following estimator

$$
\lambda_{\text {est }}=\left(N^{(T)} / N^{(0)} M_{0, T}\right)^{1 / T} .
$$

However, for a finite number of random walkers this leads to a bias. The average $\left\langle\lambda_{\text {est }}\right\rangle$ of $\lambda_{\text {est }}$ over a large number of runs of $T$ steps each can be calculated making the following observations. First, using Eq. (6), $\left\langle\lambda_{\text {est }}^{T}\right\rangle=\lambda^{T}$. Second, $\log \lambda_{\text {est }}$ has a Gaussian distribution, as guaranteed by the central limit theorem for large $T$. Denoting by $\Lambda_{0}$ and $\Sigma$ the mean and standard deviation of this Gaussian, one finds $\lambda=\exp \left(\Lambda_{0}+\frac{1}{2} T \Sigma^{2}\right)$. Up to terms negligible compared with $T \Sigma^{2}$, therefore $\Lambda_{0}=\log \left\langle\lambda_{\text {est }}\right\rangle$. This immediately yields an expression corrected for bias $\lambda=\left\langle\lambda_{\text {est }}\right\rangle+\frac{1}{2} T \sigma^{2} /\left\langle\lambda_{\text {est }}\right\rangle$, where $\sigma=\lambda \Sigma$ is the standard deviation of $\lambda_{\text {est }}$.

Alternatively, the statistical errors can be reduced by means of a modified estimator involving an approximate eigenvector for the desired energy level. ${ }^{11}$ From Eq. (6) it follows that

$$
\lambda=\frac{\sum_{k=0}^{T} \mathbf{v} \cdot \underline{H} \cdot M_{k-p, p} \mathbf{N}^{(k)}}{\sum_{k=0}^{T} \mathbf{v} \cdot M_{k-p, p} \mathbf{N}^{(k)}},
$$

for large $p$ and $T$. This, of course, is true for almost all $\mathbf{v}$, but the better $\mathbf{v}$ approximates the true ground state the greater the reduction in variance will usually be. The inner products in Eq. (8) of $\mathbf{v}$ with $\mathbf{N}^{(k)}$ and $\underline{H} \cdot \mathbf{N}^{(k)}$ are tractable, since $\mathbf{N}^{(k)}$ has only few nonvanishing components.

As a variational form of the ground state for a given quantum number $S$ of the $z$ component of the total spin, we choose, again using the representation in which the $s_{i}^{z}$ are diagonal,

$$
\mathrm{v}\left(s_{1}, \ldots, s_{n}\right)=\prod_{i=1}^{n} A\left(s_{i}, s_{i+1}\right) \delta_{s, \sum_{i=1}^{n} s_{i}},
$$

where $\underline{A}$ is a $3 \times 3$ matrix symmetric about both the diagonal and the antidiagonal. ${ }^{13}$ Consequently, $\underline{A}$ has three free parameters, not counting the overall normalization constant, and the best estimate is obtained by maximizing $\mathbf{v} \cdot \underline{H} \cdot \mathbf{v} /$ $\mathbf{v} \cdot \mathbf{v}$. For the purpose of calculating the sum over states with the restriction that $S^{z}=S$, we employ the identity

$$
\begin{aligned}
& \sum^{\prime} \\
& \quad=\frac{1}{2 n+1} \sum_{q=-n s_{1}, \ldots, s_{n}}^{n} \exp \left[2 \pi i q\left(\sum_{i=1}^{n} s_{i}-S\right) /(2 n+1)\right]
\end{aligned}
$$

where $\Sigma^{\prime}$ is the restricted sum over states. Thus, for arbitrary $n$ the Rayleigh quotient can readily be expressed in terms of the eigenvalues and eigenvectors of the matrices

$$
B_{q}\left(s_{i}, s_{i+1}\right)=e_{q}\left(s_{i}\right) A\left(s_{i}, s_{i+1}\right) e_{q}\left(s_{i+1}\right),
$$

where $e_{q}\left(s_{i}\right)=\exp \left[2 \pi i q\left(s_{i}-S / n\right) /(2 n+1)\right]$. The variational form (9) is interesting for its own sake as it can be systematically improved. ${ }^{13}$ In the Monte Carlo calculation

TABLE I. Monte Carlo results for ground-state energies $E_{0} / J$ of finite spin-1 antiferromagnetic Heisenberg chains, together with the lowest energy levels in the blocks with $S^{z}=1\left(E_{1} / J\right)$ and $S_{2}=2\left(E_{2} / J\right)$, and some numerically exact results for comparison, Refs. 3 and 10. Statistical errors in the least significant digits are shown in parentheses. Also displayed (in units of $10^{7}$ steps) are the effective lengths $T_{\mathrm{e}}$ of the runs, i.e., the target number of random walkers times the number of iterations. Top, results obtained directly from the number of random walkers. Here the target number of random walkers was 2000 . Bottom, results obtained employing a variationally approximated eigenvector with a target number of random walkers of $10^{4}$.

\begin{tabular}{ccccccc}
\hline \hline$n$ & $10^{-7} T_{\mathrm{e}}$ & \multicolumn{1}{c}{$E_{0} / J$} & $10^{-7} T_{\mathrm{e}}$ & \multicolumn{1}{c}{$E_{1} / J$} & $10^{-7} T_{\mathrm{e}}$ & $E_{2} / J$ \\
\hline 12 & 4 & $16.8713(44)$ & 4 & $16.3805(28)$ & & \\
12 & Exact & 16.8696 & Exact & 16.3854 & & \\
16 & 12 & $22.4400(39)$ & 12 & $22.0059(26)$ & 4 & $21.2946(42)$ \\
24 & 20 & $33.6326(99)$ & 20 & $33.2208(74)$ & 4 & $32.6359(94)$ \\
32 & 24 & $44.828(15)$ & 20 & $44.420(12)$ & 8 & $43.921(13)$ \\
& & & & & & \\
14 & 14 & $19.6554(11)$ & 10 & $19.1968(6)$ & 10 & $18.4214(8)$ \\
14 & Exact & 19.6551 & Exact & 19.1962 & Exact & 18.4227 \\
16 & 15 & $22.4463(14)$ & 15 & $22.0049(7)$ & 10 & $21.2906(10)$ \\
20 & 15 & $28.0453(24)$ & 15 & $27.6138(13)$ & 12 & $26.9836(15)$ \\
24 & 16 & $33.6433(38)$ & 15 & $33.2194(22)$ & 13 & $33.6483(22)$ \\
32 & 31 & $44.8497(52)$ & 24 & $44.4364(40)$ & 24 & $44.9200(29)$ \\
\hline \hline
\end{tabular}




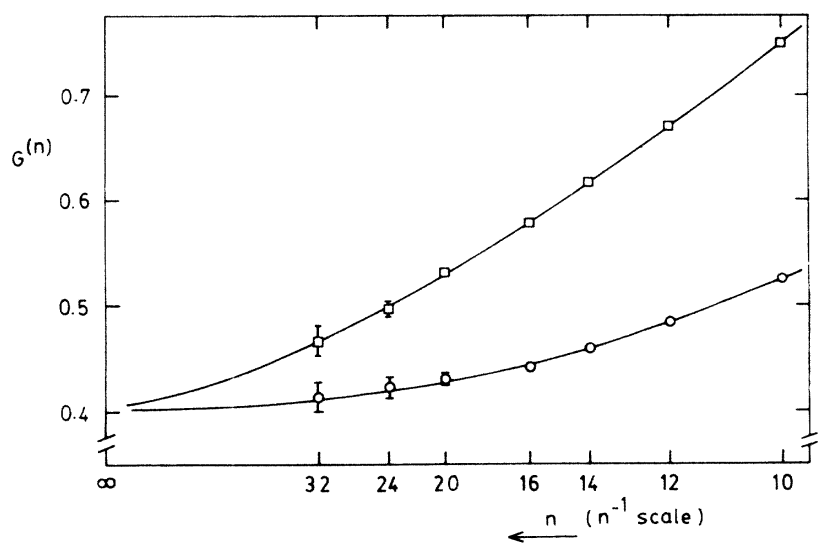

FIG. 1. Finite-system gaps $\left(E_{0}-E_{1}\right) / J$ (circles) and $\left(E_{0}-E_{2}\right) / 2 J$ (squares) vs $1 / n$. The curves are a guide to the eye. Data for $n$ up to 14 taken from Refs. 3 and 13. The error bars are twice the statistical errors as shown in Table I.

for each $\mathbf{N}^{(k)}$ we exactly calculated $\mathbf{v} \cdot \mathbf{N}^{(k)}$ and $\mathbf{v} \cdot \underline{H} \cdot \mathbf{N}^{(k)}$, in Eq. (8). Here $T$ and $p$ were chosen so large that the systematic errors due to their being finite were no longer statistically significant.

Results for various energy levels as obtained with these methods are shown in Table I. The estimates obtained for $n=12$ and $n=14$ compare well with the exact numerical results. ${ }^{3,10}$ The Monte Carlo results obtained using the (size-dependent) optimal wave functions were found to have a variance reduced roughly by an order of magnitude compared to those obtained directly from the numbers of random walkers. Comparison of the results in Table I suggests that the energy estimates in the top part of Table I possibly have a remnant (downward) bias of the same order as the statistical error. Long-time correlations between successive estimates of $\lambda$ could lead to a slight underestimation of the standard deviation $\sigma$. The resulting decrease of the calculated bias correction would produce this effect. By variation of the target number of random walkers we verified that no statistically significant bias is present in the numbers in Table I(b).

The finite-system gaps $G^{(n)}=\left(E_{0}^{(n)}-E_{1}^{(n)}\right) / J$ are plotted in Fig. 1 versus $1 / n$. The data for $n=6$ through 14 were obtained by conventional methods. ${ }^{3,10}$ The new data strongly suggest a gap $G^{(\infty)} \simeq 0.41$ for the infinite chain. No trend toward downward curvature (such as exists for spin

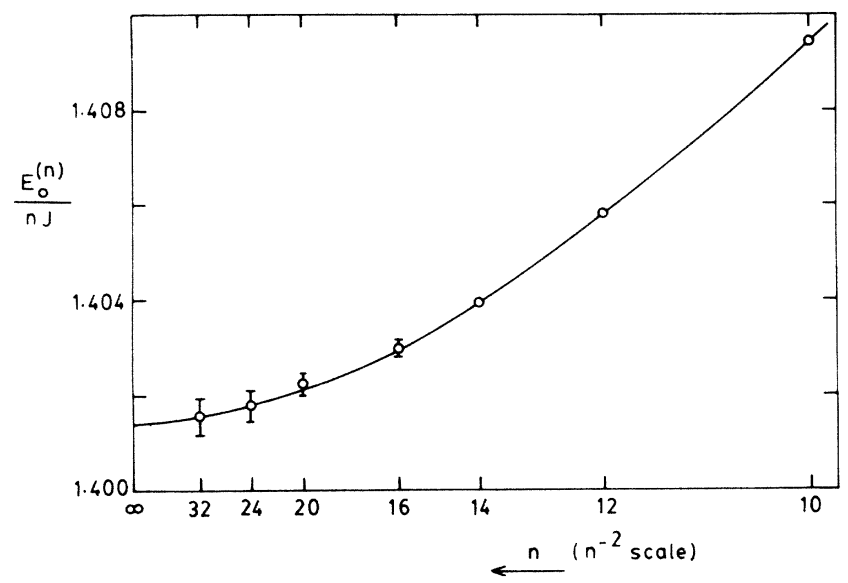

FIG. 2. Finite-system ground-state energies vs $1 / n^{2}$. Data for $n$ up to 14 taken from Refs. 3 and 11 . The error bars are twice the statistical errors as shown in Table I.

$\frac{1}{2}$ ) indicating $G^{(n)} \rightarrow 0$ for $n \rightarrow \infty$ is observable. Our conclusion, in agreement with previous finite-size calculations, is that a gap probably exists for $n \rightarrow \infty$. Another measure of the gap is the quantity $\left(E_{0}^{(n)}-E_{2}^{(n)}\right) / 2 J$, also plotted in Fig. 1. It converges more slowly than $G^{(n)}$; otherwise its behavior is similar, and extrapolates to about the same value.

Figure 2 contains the ground-state energy of the finite systems, plotted on a $1 / n^{2}$ scale. Extrapolation of the new data gives $E_{0}^{(n)} / n J=1.4015 \pm 0.0005$, in agreement with earlier work.

Note added in proof. Problems associated with having a finite number of random walkers are discussed in depth by Hetherington. ${ }^{14}$

We thank Jill C. Bonner and J. B. Parkinson for making finite-size data for $n=12$, and 14 available to us prior to publication. It is a pleasure to acknowledge helpful discussions with Jill C. Bonner, Julius Kuti, Larry McLerran, John Negele, Janos Polonyi, and Paul Rujan. This research was supported in part by the U.S. National Science Foundation under Grant No. DMR-8406186 at the University of Rhode Island, by NATO under Grant No. 198/84, by a Research Corporation Cottrell grant, and by the Dutch Stichting voor Fundamenteel Onderzoek der Materie.
${ }^{1}$ F. D. M. Haldane, Phys. Lett. 93A, 464 (1983); Phys. Rev. Lett. 50, 1153 (1983).

${ }^{2}$ C.-Y. Weng, thesis, Carnegie-Mellon University, 1968 (unpublished).

${ }^{3}$ H. W. J. Blöte, Physica B 79, 427 (1975); 93, 93 (1978).

${ }^{4}$ In the case of long-range order, the asymptotic convergence may be as slow as $1 / n$ due to interface effects, if the finite-system size is not a multiple of the size of the unit cell of the ordered state.

${ }^{5}$ R. Botet and R. Jullien, Phys. Rev. B 27, 613 (1983). See also M. Kolb, R. Botet, and R. Jullien, J. Phys. A 16, L673 (1983); R. Botet, R. Jullien, and M. Kolb, Phys. Rev. B 28, 3914 (1983).

${ }^{6}$ U. Glaus and T. Schneider, Phys. Rev. B 30, 215 (1984).

${ }^{7}$ J. C. Bonner and G. Müller, Phys. Rev. B 29, 5216 (1984; J. Appl. Phys. 55, 2395 (1984); J. Sólyom and T. A. L. Ziman, Phys. Rev. B 30, 3980 (1984).

${ }^{8}$ R. J. Baxter, J. Phys. C 6, L94 (1973).

9J. Sólyom, Phys. Rev. B 32, 7524 (1985); S. T. Chui and K. B. Ma,
Phys. Rev. B 29, 1287 (1984).

${ }^{10}$ J. B. Parkinson and J. C. Bonner, Phys. Rev. B 32, 4703 (1985).

${ }^{11}$ D. M. Ceperley and M. H. Kalos, Monte Carlo Methods in Statistical Physics, edited by K. Binder (Springer, Berlin, 1979). Also see J. Kuti, Monte Carlo Methods in Quantum Mechanical Problems, edited by M. H. Kalos, NATO Advanced Studies Institute Series B, Physics, Vol. 125 (Plenum, New York, 1984), p. 267.

${ }^{12}$ Most of the actual calculations were done with an algorithm which was slightly modified and more efficient, due to smaller fluctuations. $\underline{H}^{(1)}$ also contained off-diagonal elements, with the restriction that the sum of the elements in each column could not exceed $f_{k}$.

${ }^{13}$ R. J. Baxter, J. Stat. Phys. 19, 461 (1978). This method was formulated by Baxter for classical models in statistical mechanics. The generalization to quantum mechanical systems is straightforward.

14J. H. Hetherington, Phys. Rev. A 30, 2713 (1984). 